\title{
Estimulação neurossensorial e o seu impacto na vida de pacientes com paralisia cerebral
}

\author{
Neurosensory stimulation and the impact on the life of \\ patients with cerebral palsy
}

\section{Estimulación neurosensorial e impacto en la vida de los pacientes com parálisis cerebral}

\author{
Anna Victória de Almeida Lessa1 ${ }^{1}$, Giulia Santos Pignata², \\ Denis Carvalho Parry ${ }^{3}$
}

\begin{abstract}
1.Acadêmica do curso de Medicina. Centro Universitário de Brasília - UniCEUB, Brasília-DF, Brasil. ORCID: https://orcid.org/0000-0002-7667-1148

2.Acadêmica do curso de Medicina. Centro Universitário de Brasília - UniCEUB, Brasília-DF, Brasil. ORCID: https://orcid.org/0000-0002-5064-9575

3.Médico, Mestre, Docente do Curso de Medicina do Centro Universitário de Brasília - UniCEUB, BrasíliaDF, Brasil. ORCID: https://orcid.org/0000-0003-1258-3285
\end{abstract}

\begin{abstract}
Resumo
Introdução. A paralisia cerebral (PC) é uma patologia multifatorial, complexa e crônica, que ocorre durante a infância e se prolonga ao longo da vida, tendo como base o comprometimento no desenvolvimento psicomotor e neurossensorial, levando a limitações secundárias que afetam a qualidade de vida dos pacientes. Objetivo. Avaliar o impacto da estimulação neurossensorial na qualidade de vida de pacientes com paralisia cerebral. Método. Pesquisa documental referente a um estudo de caso clínico de caráter qualitativo e retrospectivo, entre janeiro de 2016 e dezembro de 2020. Resultados. O suporte terapêutico ofertado deve englobar o apoio familiar juntamente com uma equipe multidisciplinar, que irão atuar na mobilização precoce, controle motor e postural, incentivo linguístico, sensorial e social. Desta forma, as técnicas de estimulação neurossensorial vêm sendo estudadas e empregadas, a fim de avaliar a resposta dos pacientes e suas perspectivas promissoras. Conclusão. As técnicas de estimulação neurossensorial que atuam nos cincos sentidos além de atividades que melhoram a função motora, ainda não possuem protocolos específicos de avaliação para pacientes com PC. No entanto é notório que as modificações a serem alcançadas com essas metodologias ocorrem de forma gradual, com grande impacto na qualidade de vida desses indivíduos, principalmente com o apoio e cuidado integrado da família com uma equipe multiprofissional.
\end{abstract}

Unitermos. infância; comprometimento neuropsicomotor; estimulação neurossensorial; suporte multidisciplinar; paralisia cerebral

\footnotetext{
Abstract

Introduction. Cerebral palsy (CP) is a multifactorial, complex and chronic pathology that occurs during childhood and is prolonged throughout life, based on the impairment of psychomotor and sensorineural development, leading to secondary limitations that affect the quality of life of patients. Objectives. Evaluate the impact of sensorineural stimulation on the quality of life of patients with cerebral palsy. Method. Documentary research referring to a clinical case study of a qualitative and retrospective nature, between January 2016 and December 2020. Results. The therapeutic support offered must include family support together with a multidisciplinary team, which will act in early mobilization, motor and postural control, linguistic, sensorial and social encouragement. Thus, sensorineural stimulation techniques have been studied and used in order to assess the response of patients and their promising prospects. Conclusions. Sensorineural stimulation techniques that act on the five senses, in addition to activities that improve motor function, do not yet have specific assessment protocols for patients with CP. However, it is clear that the changes to be achieved
} 
with these methodologies occur gradually, with a great impact on the quality of life of these individuals, especially with the support and integrated care of the family with a multidisciplinary team.

Keywords. childhood; neuropsychomotor impairment; sensorineural stimulation; multidisciplinary support; cerebral palsy

\begin{abstract}
Resumen
Introducción. La parálisis cerebral $(P C)$ es una patología multifactorial, compleja y crónica que se presenta durante la infancia y se prolonga a lo largo de la vida, basada en el deterioro del desarrollo psicomotor y neurosensorial, lo que genera limitaciones secundarias que afectan la calidad de vida de los pacientes. Metas. Evaluar el impacto de la estimulación neurosensorial en la calidad de vida de los pacientes con parálisis cerebral. Método. Investigación documental referida a un estudio de caso clínico de carácter cualitativo y retrospectivo, entre Enero de 2016 y Diciembre de 2020. Resultados. El apoyo terapéutico ofrecido debe abarcar el apoyo familiar junto con un equipo multidisciplinar, que actuará en la movilización temprana, el control motor y postural, el estímulo lingüístico, sensorial y social. Así, se han estudiado y utilizado técnicas de estimulación neurosensorial para evaluar la respuesta de los pacientes y sus promisorias perspectivas. Conclusiones. Las técnicas de estimulación neurosensorial que actúan sobre los cincos sentidos, además de las actividades que mejoran la función motora, aún no cuentan con protocolos de valoración específicos para pacientes con PC. Sin embargo, es evidente que los cambios que se van a conseguir con estas metodologías se producen de forma paulatina, con un gran impacto en la calidad de vida de estos individuos, especialmente con el apoyo y cuidado integral de la familia con un equipe multidisciplinario.
\end{abstract}

Palabras clave. infancia; deterioro neuropsicomotor; estimulación neurosensorial; apoyo multidisciplinario; parálisis cerebral

Trabalho realizado no Centro Universitário de Brasília - UniCEUB, Brasília-DF, Brasil.

Conflito de interesse: não

Recebido em: 22/08/2021

Aceito em: 05/10/2021

Endereço para correspondência: Denis Carvalho Parry. Centro Universitário de Brasília - UniCEUB. SEPN 707/907. Campos Universitário. CEP 70790-075. Brasília-DF, Brasil. Email: denisparry@hotmail.com

\title{
INTRODUÇÃO
}

A conceituação sobre paralisia cerebral (PC) envolve o entendimento sobre modificações heterogêneas no neurodesenvolvimento psicomotor que acomete principalmente a faixa etária infantil, tendo o termo surgido historicamente em 1897 com Sigmund Freud, ao avaliar recém-nascidos com complicações durante o parto e nascimento ${ }^{1}$.
A PC é
uma
patologia complexa,
sendo necessário

subdividi-la de acordo com o parâmetro de avaliação, a fim de diagnosticar as crianças e oferecer tratamento, levando- 
se em conta a limitação funcional, motora e neurossensorial, como também comorbidades associadas e fatores de riscos genéticos e adquiridos. Assim, percebe-se que a morbimortalidade sofre variações, juntamente com o nível de suporte terapêutico ofertado, e desta forma, o risco é proporcional ao grau e ao número de deficiências notadas ${ }^{2}$.

A PC espástica é o subtipo mais frequente, tendo como foco de acometimento o primeiro neurônio motor e, consequentemente, um quadro clínico característico de síndrome piramidal, se associando com hipertonia e reflexos tendinosos profundos intensificados, o que leva à fraqueza muscular, comprometimento da marcha e da postura ${ }^{3,4}$.

O segundo subtipo mais encontrado nos pacientes é a PC discinética, a qual está associada ao comprometimento da funcionalidade dos neurônios presentes nos gânglios da base $^{4}$. Tendo como diferencial a interferência de fatores ambientais nas manifestações clínicas, tais como: estresse, irritabilidade e desgaste físico ao longo do dia; tendo em vista que o comprometimento motor é característico de uma Síndrome Extrapiramidal5,6. Além disso, é uma condição neurológica que pode cursar com duas variáveis motoras: distônica e coreatetoide; a distonia é percebida na maioria dos pacientes, tendo como característica movimentos e posturas anômalas, devido à ação de grupamentos musculares que geram contrações involuntárias. Enquanto a coreoatetose se estabelece pela alternância entre movimentos rápidos, bruscos e involuntários (coreia) com movimentos lentos e contorcidos (atetose) ${ }^{6}$. 
Por fim deve-se citar a PC atáxica que tem como alvo de lesão o cerebelo, e consequentemente, manifestações associadas a distúrbios de coordenação da função muscular, o que leva à realização de movimentos com falência de força, precisão e sincronia necessárias ${ }^{2}$.

Tendo como enfoque a avaliação neuropsicomotora realizada durante a infância, o diagnóstico de PC é clínico, com o uso de exames complementares para a exclusão de diagnósticos diferenciais, se necessário. O princípio mais importante é compreender que uma criança com PC deve ser avaliada de forma holística, levando em conta o contexto social e familiar que está inserida, e principalmente, o acompanhamento médico periódico para se ter efetividade nas medidas terapêuticas a serem adotadas ${ }^{2}$.

Desta forma, o tratamento envolve um suporte com equipe multidisciplinar, tendo em vista ser uma patologia multifatorial crônica não progressiva, que necessita de medidas ativas, tais como: fisioterapia, terapia ocupacional e neurossensorial, mobilização com suporte de aparelhos ortopédicos, além de intervenções farmacológicas ${ }^{7}$.

Tendo como entendimento que o suporte terapêutico na PC deve compreender o indivíduo, como já citado, de forma holística, nota-se o incentivo a estudos que buscam avaliar a efetividade da estimulação neurossensorial nesse espectro, visto a alta prevalência de distúrbios secundários sensoriais, linguísticos, cognitivos, emocionais e sociais nos pacientes, que vão além do acometimento neuropsicomotor. Percebese que pode ser uma consequência direta da PC como 
também da falta de estímulos a experiências ao longo da vida. Assim, a equipe multidisciplinar deve-se voltar a medidas que estimulem as funções: auditiva, visual, função motora, função manual, habilidades cognitivas e sociais, comunicação e linguagem, além da motricidade orofacial ${ }^{8}$.

Por ser um campo que ainda está sendo estudado, percebe-se que não há padronização de testes que possam avaliar o comprometimento neurossensorial na PC, e consequentemente, não há evidências suficientes quer permitam avaliar variações no processamento e atraso das habilidades manipulativas, cognitivas e da percepção visuoespacial em cada paciente. No entanto, há perspectivas promissoras que demonstram aumento da qualidade de vida dos portadores de PC que foram expostos a terapias que atuem na função tátil, proprioceptiva e vestibular ${ }^{9}$.

Portanto, devido a significativa prevalência e o comprometimento funcional por toda a vida relacionados à PC, o presente estudo faz-se importante para avaliar como a estimulação neurossensorial impacta nos aspectos físicos, cognitivos e afetivos em uma paciente do sexo feminino, de 19 anos de idade com PC, com ênfase na melhoria da qualidade de vida desta e comparar com dados da literatura.

\section{MÉTODO}

O estudo foi iniciado após aprovação do Comitê de Ética (CEP) do Centro Universitário de Brasília - UniCEUB, de Brasília/DF, sob parecer de número 4.872.129 (CAEE: 46479121.0.0000.0023). Trata-se de uma pesquisa 
documental referente a um estudo de caso clínico de caráter qualitativo e retrospectivo, entre janeiro de 2016 e dezembro de 2020, tendo como amostra uma paciente do sexo feminino, de dezenove anos de idade, com diagnóstico de paralisia cerebral espástica desde o nascimento, tendo como complicação a leucomalácia periventricular. A análise documental foi realizada através da avaliação retrospectiva dos prontuários, em posse da família e disponibilizados para a pesquisa, dos anos de 2016 a 2020. A participante deveria obedecer ao critério de diagnóstico de paralisia cerebral em uso de terapia neurossensorial; sem ter critério de exclusão designado. Os pais/responsáveis assinaram o Termo de Consentimento Livre e Esclarecido (TCLE).

\section{RESULTADOS}

Paciente, do sexo feminino, de 19 anos de idade, com diagnóstico de paralisia cerebral espástica, tendo como complicação a leucomalácia periventricular.

Mãe com histórico gestacional de gestação gemelar monocoriônica-diamniótica, com diagnóstico de síndrome de transfusão feto-fetal com 24 semanas de idade gestacional, aos 37 anos de idade. O quadro evoluiu para parto prematuro com 32 semanas de idade gestacional, devido à diminuição acentuada do volume de líquido amniótico, acompanhada de centralização dos fluxos em ambos os fetos, sendo então indicada interrupção imediata da gestação em face das evidências de sofrimento fetal. 
Ao nascer, após ressuscitação cardiopulmonar e a posterior realização de exames na sala de parto, as recémnascidas (RNs) gemelares, ambas do sexo feminino, foram encaminhadas para a Unidade de Terapia Intensiva Neonatal (UTIN). A primeira gemelar apresentou 800 gramas ao nascer, Apgar 5 e 7, tendo como diagnósticos: gemelaridade, doença da membrana hialina, persistência do canal arterial, pneumotórax, apneia do sono, infecção inespecífica perinatal, pé torto congênito e paquigiria. A segunda gemelar, com peso de 1360 gramas ao nascer apresentou Apgar 5 e 8, comprimento de $40 \mathrm{~cm}$ e perímetro cefálico de $28 \mathrm{~cm}$ teve como diagnósticos: gemelaridade (maior gemelar discordante), asfixia perinatal, doença da membrana hialina, pneumotórax à direita, hidrocefalia supratentorial e encefalomalacia em lobos frontais e parietais bilateralmente.

O diagnóstico de paralisia cerebral foi realizado aos 60 dias de vida na primeira gemelar e aos 30 dias de vida na segunda gemelar. A primeira gemelar, posteriormente, evoluiu com óbito aos 2 anos e 8 meses, após diagnóstico de pneumonia e posteriores complicações durante o tratamento realizado em ambiente hospitalar.

A segunda gemelar iniciou o acompanhamento multiprofissional pediátrico, neurológico, de terapia ocupacional, com avaliações auditivas e visuais periódicas. Fundoscopia realizada aos 10 meses de vida pelo oftalmologista demonstrou derrame ocular, mas com a 
presença dos reflexos fotomotores e sem outras alterações dignas de nota.

Durante o processo de crescimento e desenvolvimento, a criança não apresentou controle postural, tônus cervical, nem capacidade de sentar sem apoio ou posterior desenvolvimento da marcha. A alimentação era realizada por via oral, por meio de alimentos pastosos e em livre demanda, no entanto sem ganho de peso adequado para a idade. Após estímulos realizados pela família e pela equipe multidisciplinar composta por fonoaudióloga, fisioterapeuta, neurologista, pediatra e terapeuta ocupacional, a paciente desenvolveu capacidade de controle da marcha com apoio, resposta aos estímulos por meio da emissão de sons e do reflexo pupilar voluntário, além da protrusão da língua ao desejar determinados alimentos. Ressalta-se, no entanto que a criança com o avanço da idade, evoluiu com comprometimento motor em membros superiores e inferiores, característico da paralisia cerebral espástica, além de intensa atrofia, rigidez muscular e cifoescoliose.

No que diz respeito à visão e ao tato, a paciente não desenvolveu a capacidade de manipular objetos, estereognosia e acuidade espacial, enquanto na visão, notou-se diminuição da acuidade visual, com baixa resposta ao reflexo pupilar involuntário e consensual, mas com capacidade de concentrar o olhar em direção aos objetos ou faces.

Aos 15 anos de idade, a paciente apresentou diagnóstico de pneumonia comunitária, sendo então 
encaminhada para o serviço hospitalar na cidade de Brasília. Durante a internação na Unidade de Terapia Intensiva (UTI), evoluiu ao longo dos 45 dias com franco desconforto respiratório, pneumotórax, hemotórax e parada cardiorrespiratória durante 11 minutos, infecção hospitalar e realização de traqueostomia. Após receber alta iniciou 0 acompanhamento pela equipe do home care, com dano cerebral mais avançado, sem resposta aos estímulos sensoriais anteriormente desenvolvidos, além de alimentação por sonda nasoenteral.

Durante os cinco anos de acompanhamento entre 20162020 após receber alta hospitalar, a paciente apresentou quadros repetidos de pneumonia nosocomial com necessidade de ventilação mecânica, além da realização da cirurgia de gastrostomia para ganho ponderal. Atualmente, respira sem ajuda de suporte ventilatório por oxigênio, mas com a permanência da traqueostomia e com gastrostomia implantada.

Foram analisados os prontuários buscando-se compreender as técnicas utilizadas para estimulação neurossensorial na paciente descrita, nesse período dos últimos cinco anos e com isso avaliar a validade das mesmas no que diz respeito às respostas apresentadas pela paciente:

Estímulo olfativo - feito por meio de substâncias com odores característicos, próximas às narinas, a fim de aumentar a variedade de odores reconhecíveis e a demonstração de expressões faciais características, de 
acordo com cada uma das substâncias testadas. Durante os anos avaliados apresentou a seguinte evolução cronológica:

Ano 0 (2016) - presença de sonda nasoenteral que dificultava a realização de estímulos olfativos.

Ano 1 (2017) - retirada da sonda com início de testes com substâncias de odores característicos, sem resposta aos estímulos.

Ano 2 (2018) - início da resposta a determinadas substâncias colocadas próximas às narinas, principalmente com indução de espirros.

Ano 3 (2019) - aumento da eficácia do reconhecimento dos odores testados, com resposta da protusão da língua de acordo com o desejo em provar a substância.

Ano 4 (2020) - desenvolvimento de expressões faciais características de acordo com os odores reconhecíveis, além da movimentação ocular responsiva com aceitação ou repulsa à substância ofertada.

Estímulo tátil - foi testado por meio do emprego de materiais com diferentes superfícies, em contato com a pele da paciente, podendo-se citar: argila, massa de modelar, bola, brinquedos, grãos, entre outros. A resposta foi observada de forma gradual, percebendo- se a evolução no tato discriminatório, com expressões faciais variáveis de acordo com o aspecto do objeto a ser tocado, sendo notória uma expressão facial de tranquilidade quando os membros superiores ou inferiores entravam em contato com áreas lisas e homogêneas, enquanto objetos com características rugosas e ásperas eram vistos com fácies de repulsa. Além 
disso, o incentivo à escrita e à pintura com o uso de pinceis coloridos e chamativos, como também o contato com instrumentos musicais, ao longo dos anos, demonstrou-se maior coordenação motora e foco nas atividades realizadas, com aumento do tônus cervical e equilíbrio postural, na seguinte cronologia:

Ano 0 (2016) - ausência de reatividade no contato com objetos à superfície cutânea e incoordenação motora e inefetividade do equilíbrio postural.

Ano 1 (2017) - permanência da ausência de reatividade no contato de objetos e incoordenação motora e inefetividade do equilíbrio postural.

Ano 2 (2018) - início da demonstração de expressões faciais diversas de acordo com as características das substâncias a serem tocadas e incoordenação motora e inefetividade do equilíbrio postural.

Ano 3 (2019) - evolução do tato discriminatório com fácies de reatividade características e início da coordenação motora sem controle postural.

Ano 4 (2020) - capacidade de segurar objetos por alguns segundos e expressar reatividade ao entrar em contato com a pele e melhor coordenação motora e início de equilíbrio postural.

Estímulo gustativo - realizaram-se atividades com alimentos de diferentes sabores, a fim de testar e incentivar o reconhecimento do azedo, amargo, doce e salgado, com o uso de pequenas quantidades de substâncias em contato com a língua da paciente. Desta forma, gradualmente 
percebeu-se início do processo de sucção, além da demonstração de expressões faciais características para cada sabor, notando-se maior desejo e satisfação ao entrar em contato com alimentos de características doces. Outras medidas realizadas pela equipe multidisciplinar para estimular a sucção foi o uso de chupeta com pequenas quantidades de líquidos com diversos sabores, como também atividades realizadas pela fonoaudióloga para redução da rigidez da musculatura temporomandibular. Além disso, é importante ressaltar a substituição da alimentação industrializada para a caseira ofertada por via de gastrostomia, com maior ganho de peso para paciente e melhora da qualidade de vida. Evolução cronológica:

Ano 0 (2016) - sonda nasoenteral para oferta de alimentos e ganho ponderal, sem estímulos gustativos.

Ano 1 (2017) - substituição da sonda nasoenteral para gastrostomia com oferta de alimentos industrializados, sem estímulos gustativos.

Ano 2 (2018) - início das atividades com a equipe de fonoaudiologia para melhora da sucção e redução da rigidez da musculatura temporomandibular, sem estímulos gustativos.

Ano 3 (2019) - mudança da alimentação industrializada para caseira ofertada por via de gastrostomia.

Ano 4 (2020) - início de terapias com incentivo ao contato da língua da paciente com diferentes sabores e fácies características para cada substância, sendo notado a consistência da repulsa à alimentos amargos e azedos. 
Estímulo visual - técnicas de aprimoramento e fixação visual foram utilizadas por meio de placas com cores primárias chamativas, luzes, além de imagens atrativas em direção ao campo de visão, sendo notória a melhor fixação do olhar e o seu desvio de acordo com a mudança das imagens, com a seguinte evolução:

Ano 0 (2016) - paciente inerte, sem movimentação ocular reativa à qualquer técnica realizada próxima ao seu campo visual.

Ano 1 (2017) - ausência de movimentação ocular para qualquer técnica realizada próxima ao seu campo visual.

Ano 2 (2018) - início da procura com o olhar a imagens próximas ao seu campo de visão.

Ano 3 (2019) - movimentação ocular responsiva com desvio de acordo com as variações das imagens no campo visual.

Ano 4 (2020) - busca ativa com a movimentação ocular a imagens dentro do campo de visão, com fixação do olhar correspondente.

Estímulo auditivo - utilizou-se de musicoterapia, com músicas de ópera para auxiliar na atenção, relaxamento e audição, além de outros estímulos sonoros tais como sons de animais e o som do mar, notando-se maior fixação do olhar e procura do som produzido no ambiente ao longo dos anos de observação. Ao longo dos anos, assim evoluiu:

Ano 0 (2016) - ausência da capacidade de concentração e não reconhecimento de sons. 
Ano 1 (2017) - início do reconhecimento das vozes emitidas pelos familiares, sem busca ativa com movimentação ocular ou cervical.

Ano 2 (2018) - início da busca ativa dos sons com movimentação do globo ocular, ausência de movimentação cervical e capacidade de expressar fácies de emoção ou reatividade.

Ano 3 (2019) - início da busca ativa dos sons com movimentação ocular e cervical.

Ano 4 (2020) - reconhecimento de sons com movimentação rápida ocular e cervical, capacidade de se concentrar e oscilações de humor de acordo com o estilo musical.

\section{DISCUSSÃO}

Apesar do comprometimento sensorial e funcional secundários à $\mathrm{PC}$, $\mathrm{O}$ desenvolvimento de técnicas de estimulação neurossensorial realizadas de forma precoce contribuem para benefícios intelectuais, físicos e afetivos, e assim, ao aumento da qualidade de vida dos pacientes com maior adaptação ao meio em que vivem ${ }^{10}$.

Estudos demonstram que crianças com PC possuem diferentes graus de comprometimento da manipulação de objetos, de acordo com o subtipo associado. Assim, notouse o maior comprometimento da estereognosia, capacidade de reconhecer a forma do objeto por meio do tato; e a acuidade espacial, o que levam a menor destreza na função somatossensorial dos membros superiores. No entanto, 
ainda não há testes padronizados que possam avaliar a função tátil nos pacientes de forma homogênea e sem variações significativas no resultado da análise; há estudos que demonstraram a tentativa dos exames de discriminação de dois pontos, mas sem chegar a uma conclusão efetiva ${ }^{11}$.

Do ponto de vista do comprometimento auditivo, notase o uso de música como um método clínico-terapêutico, com finalidade de aprimoramento cognitivo, físico, mental e social positivos. Tendo sua ação por meio dos componentes de som, ritmo, melodia e harmonia ${ }^{12}$.

O estudo sobre a estimulação auditiva rítmica (RAS), segundo KWAK, ainda precisa de maior aprofundamento na análise em pacientes com PC. No entanto, o estudo demonstra que a partir da RAS, técnica que utiliza os efeitos do ritmo auditivo no sistema cerebral motor, e assim, a música como forma de regulação do movimento corporal; os pacientes com PC espástica podem sincronizar o corpo aos movimentos de forma rítmica, por meio do treinamento auditivo. Desta forma, o estudo demonstrou uma possibilidade de medida terapêutica adjuvante à fisioterapia, tendo em vista o comprometimento variável da marcha, postura e equilíbrio corporal nos pacientes com PC. Ressaltase, no entanto, a necessidade de maiores pesquisas para entender como é o mecanismo de ação da RAS na postura corporal e na estabilidade das crianças com PC ${ }^{13}$.

Além da disfunção auditiva e tátil, é importante frisar o comprometimento do paladar nas crianças com PC. Sendo significativa a presença de distúrbios alimentares, tais como: 
disfagia, disfunção oromotora, refluxo gastroesofágico, discinesia faringoesofágica e aversão do apetite ${ }^{14}$. Devido aos subtipos de PC, há graus variáveis de comprometimento neuropsicomotor, e assim, há variação na gravidade dos distúrbios do paladar. As principais consequências são: broncoaspiração e possíveis complicações pulmonares, déficit nutricional, hidratação inadequada, retardo no crescimento, refeições mais longas, além de uma evolução mais lenta na variedade de textura dos alimentos para a ingestão e deglutição ${ }^{14,15}$.

Há uma gama de distúrbios orofaríngeos que podem ser percebidos no espectro de pacientes com PC, que leva à disfunção oromotora. Arvedson descreve: "fechamento labial reduzido, função inadequada da língua, reflexo exagerado de mordida, hipersensibilidade tátil, retardo no início da deglutição, aumento da produção de saliva (baba) e motilidade faríngea menos eficaz"15. A disfagia na PC, por sua vez, tem como causa um distúrbio no sistema nervoso central (SNC), que leva à variação no tônus passivo, tônus ativo normal e reflexos primitivos intensos e persistentes. $O$ comprometimento na deglutição de líquidos relaciona-se ao retardo no início da deglutição; enquanto na disfagia por sólidos, há variação de acordo com o grau de motilidade da orofaringe, o que desencadeia a ingestão de alimentos espessos ou pastosos ${ }^{15}$.

Desta forma, o incentivo ao estímulo sensorial com uma equipe interdisciplinar ajuda o paciente com PC, de acordo com suas necessidades, tratar e amenizar os distúrbios 
alimentares e a disfagia orofaríngea. As medidas terapêuticas, por sua vez, devem focar em promover vias aéreas pérvias associadas ao suporte calórico, hidratação e função pulmonar adequados. Assim, o objetivo final será promover um paladar com o mínimo de prazer para a criança, além de contribuir para o manejo da função secretiva, deglutição e preservação da cavidade oral ${ }^{15}$. Tendo como consequência final a redução da morbidade e da taxa de hospitalizações ${ }^{14}$.

Dentre as técnicas que podem ser elucidadas no âmbito da estimulação sensorial, deve-se ressaltar o incentivo às habilidades orais, que vão evoluindo de acordo com a idade e complexidade alimentar. Portanto, indica-se o uso de meios de sucção, como as chupetas, em recém-nascidos; e com o avançar da idade, de acordo com a capacidade de alimentação por via oral, o incentivo à ingestão de pequenas quantidades de alimentos, sejam líquidos ou sólidos, a fim de aumentar a variedade do paladar, principalmente em crianças em uso de gastrostomia ${ }^{15}$.

Por fim, a gastrostomia é uma forma muito usada de suporte nutricional nos pacientes com PC, de acordo com a gravidade do comprometimento neuropsicomotor e da capacidade de deglutição ${ }^{15}$. Tendo em vista que são crianças que possuem oferta energética reduzida e imobilidade, que contribuem para os déficits nutricionais associados ${ }^{14}$.

O olfato é outro sentido básico que pode ser afetado em crianças com PC. Sendo notória a sua significância para o aporte nutricional, além de promover segurança e melhora 
da qualidade de vida desses pacientes. Contudo, a capacidade de identificação de odores depende da experiência adquirida ao longo da vida, além da percepção e cognição. E assim, percebe-se a escassez de estudos que demonstram, de forma aprofundada, o comprometimento olfativo na $\mathrm{PC}^{16}$.

Um estudo realizado em pacientes com PC demonstrou que há o comprometimento visual em mais de $80 \%$ dos casos. Ressaltando-se alteração visual e defeitos oculomotores, tendo como principais diagnósticos os erros de refração (ametropia) e estrabismo; sendo rara a presença de doenças oculares ${ }^{17}$. Outro estudo descrito por Da Costa, associa o grau de comprometimento motor, de acordo com - Sistema de Classificação da Função Motora Grossa (GMFCS), com a perda da acuidade visual nas crianças com PC. Uma técnica utilizada, de forma complementar, para avaliação clínica foi o Potencial Evocado Visual de Varredura (VEP), que mede a acuidade visual exposta a estímulos, com maior rapidez e sem a necessidade de intervalos longos de atenção e foco visual, de acordo com frequências espaciais presentes no ambiente. Essa ferramenta demonstrou a importância de experiências sensoriais vivenciadas diariamente, como meio de expandir a entrada de estímulos no córtex visual associativo ${ }^{18}$.

Além disso, a visão possui seu papel na capacidade de buscar ou fixar o olhar aos objetos, além de atuar juntamente com o sistema vestibular no controle fino dos movimentos da cabeça, tendo ação na manutenção da 
postura e do equilíbrio ${ }^{19,20}$. Alguns estudos demonstram que crianças com PC, de acordo com o subtipo e com a gravidade de comprometimento, possuem dificuldade na manutenção do tônus cervical durante atividades dinâmicas, o que pode estar relacionado a padrões inadequados de atividade muscular truncal 20. Portanto, estímulos sensoriais que atuam no treinamento de fixação visual e na função perceptivo-motora podem ajudar esses pacientes ${ }^{19,20}$.

\section{CONCLUSÃO}

Os estudos acerca da eficácia das técnicas de estimulação neurossensorial que atuam nos cinco sentidos (olfato, paladar, tato, audição e visão), além de atividades que melhoram a função motora ainda são recentes e não há protocolos específicos de avaliação que possam estimar, em valores estatísticos, a efetividade de cada técnica em pacientes com PC. No entanto, é importante ressaltar que devido ser uma patologia complexa, crônica, irreversível e multifatorial, deve-se avaliar individualmente cada paciente de acordo com o seu comprometimento cerebral, motor e neurossensorial, como também, frisar que as modificações a serem alcançadas com as metodologias de estímulo podem ocorrer de forma gradual, ao longo dos anos, mas com grande impacto na qualidade de vida dessas crianças.

A análise realizada, ao longo de cinco anos, sobre a análise documental apresentada de uma paciente de dezenove anos com PC espástica desde o nascimento, demonstra como o apoio e cuidado integrado da família com 
uma equipe multidisciplinar que utiliza técnicas neurossensoriais, principalmente se realizadas de forma precoce, podem impactar na qualidade de vida à longo prazo.

Podendo-se ressaltar nesta paciente alguns avanços alcançados, tais como: aumento do peso corporal, do tônus cervical e do controle postural; melhora da função motora e da fixação do olhar com busca ativa a objetos e a familiares dentro do campo visual; evolução no tato discriminatório e na expressão de fácies características de acordo com a emoção causada por determinados estímulos, como por exemplo, uma substância com odor característico.

Essas evoluções alcançadas demonstram que a PC representa uma área vasta e ainda pouco estudada dentro do meio científico, mas que necessita cada vez mais de análises que possam ajudar e discriminar técnicas e metodologias a serem realizadas que melhoram a função neurossensorial e motora e, assim, promover maior conforto e bem-estar ao longo da vida desses pacientes.

Reforçamos que sem o envolvimento atento e paciente da família, sempre buscando informações junto à equipe multidisciplinar e participação ativa no processo, os resultados estariam fadados a não alcançar toda a evolução favorável que um paciente portador de PC tem o potencial de atingir.

\section{REFERÊNCIAS}

1.Baxter P, Morris C, Rosenbaum P, Paneth N, Leviton A, Goldstein M, et al. The definition and classification of cerebral palsy. Dev Med Child Neurol 2007;49:1-44.

https://doi.org/10.1111/j.14698749.2007.00001.x 
2.O'Shea TM. Diagnosis, treatment, and prevention of cerebral palsy in near-term/term infants. Clin Obstetr Gynecol 2008;51:816-28. https://doi.org/10.1097/GRF.0b013e3181870ba7

3.Bar-On L, Molenaers G, Aertbeliën E, Campenhout AV, Feys H, Nuttin $B$, et al. Spasticity and its contribution to hypertonia in cerebral palsy. BioMed Res Int 2015;2015. https://doi.org/10.1155/2015/317047

4.Sanger TD, Delgado MR, Gaebler-Spira D, Hallett M, Mink JW. Classification and definition of disorders causing hypertonia in childhood. Pediatrics 2003;111:89-97. https://doi.org/10.1542/peds.111.1.e89

5.Pakula AT, Braun KVN, Yeargin-Allsopp M. Cerebral palsy: classification and epidemiology. Phys Med Rehab Clin 2009;20:425-52. https://doi.org/10.1016/j.pmr.2009.06.001

6. Monbaliu E, Himmelmann K, Lin JP, Ortibus E, Bonouvrié L, Feys $H$, et al. Clinical presentation and management of dyskinetic cerebral palsy. Lancet Neurol 2017;16:741-9. https://doi.org/10.1016/S14744422(17)30252-1

7.Koman LA, Smith BP, Shilt JS. Cerebral palsy. Lancet 2004;363:1619-31. https://doi.org/10.1016/S0140-6736(04)16207$\underline{7 .}$

8. Ministério da Saúde, Secretaria de Atenção a Saúde. Diretrizes de Atenção à Pessoa com Paralisia Cerebral, 2013 (endereço na internet) (acessado em: 03/2020). Disponível em: https://bvsms.saude.gov.br/bvs/publicacoes/diretrizes atencao paral isia cerebral.pdf

9. Mayston MJ. People with cerebral palsy: effects of and perspectives for therapy. Neural Plasticit 2001;8:51-69. https://doi.org/10.1155/NP.2001.51

10.Ministério da Saúde. Secretaria de Atenção à Saúde. Diretrizes de estimulação precoce: crianças de zero a 3 anos com atraso no desenvolvimento neuropsicomotor decorrente de microcefalia; 2016 (endereço na internet). (acessado em: abril/2020). Disponível em: https://bvsms.saude.gov.br/bvs/publicacoes/diretrizes estimulacao c riancas 0a3anos neuropsicomotor.pdf

11.Wingert JR, Burton $\mathrm{H}$, Sinclair RJ, Brunstrom JE, Damiana DL. Tactile sensory abilities in cerebral palsy: deficits in roughness and object discrimination. Develop Med Child Neurol 2008;50:832-8. https://doi.org/10.1111/j.1469-8749.2008.03105.x

12. Correa AGD, Ficheman IK, Nascimento M, Lopes RD. Computer assisted music therapy: A case study of an augmented reality musical system for children with cerebral palsy rehabilitation. Proceedings of the 2009 Ninth IEEE International Conference on Advanced Learning Technologies; 15-17 July 2009; Riga, Latvia. p.218-20. https://doi.org/10.1109/ICALT.2009.111

13.Kwak EE. Effect of rhythmic auditory stimulation on gait performance in children with spastic cerebral palsy. J Mus Therap 2007;44:198-216. https://doi.org/10.1093/jmt/44.3.198

14.Schwarz SM, Corredor J, Fisher-Medina J, Cohen J, Rabinowitz S. Diagnosis and treatment of feeding disorders in children with 
developmental disabilities. Pediatrics 2001;108:671-6. https://doi.org/10.1542/peds.108.3.671

15. Arvedson JC. Feeding children with cerebral palsy and swallowing difficulties. Eur J Clin Nut 2013;67:9-12. https://doi.org/10.1038/ejcn.2013.224

16.Dan B. The importance of olfaction in neurodisability. Develop Med Child Neurol 2019;61:4. https://doi.org/10.1111/dmcn.14075

17. Lo Cascio GP. A study of vision in cerebral palsy. Optom Vision Sci 1977;54:332-7. http://doi.org/10.1097/00006324-197705000-00011 18. Costa MF, Salomão SR, Berezovsky A, Haro FM, Ventura DF. Relationship between vision and motor impairment in children with spastic cerebral palsy: new evidence from electrophysiology. Behav Brain Res 2004;149:145-50. https://doi.org/10.1016/S01664328(03)00223-7

19.Duckman RH. Vision therapy for the child with cerebral palsy. J Am Optom Assoc 1987;58:28-35. https://psycnet.apa.org/record/1988$14445-001$

20.Saavedra S, Woollacott M. Donkelaar PV. Head stability during quiet sitting in children with cerebral palsy: effect of vision and trunk support. Exp Brain Res 2010;201:13-23. https://doi.org/10.1007/s00221-009-2001-4 